\title{
Study on the Kinematics and Operating Space of a Foreign Body Salvage Robot at the Bottom of the Reactor Pool
}

\author{
Zhao Wang, Lingyu Sun* and Yaiqiao Zhu \\ School of Mechanical Engineering, Hebei University of Technology, Tianjin Hongqiao District Dingzigu One Road Street, China \\ ${ }^{*}$ Corresponding author
}

\begin{abstract}
Nuclear power plant inspection underwater crawling robot can replace foreign operation and maintenance personnel to complete the reactor core and pool fishing operation. In this paper, through the analysis and research on the configuration and control system of the robot, based on kinematics model of underwater crawling robot, analyzing its kinematics , thus establishing kinematics and quasi solution of the equations for the robot, through surface equation, geometric equation and kinematics equation of each link has been established manipulator and constraint plane combination, calculate the maximum ground crawl space boundary, Motion module using SolidWorks to simulate the boundary track verification, finally through the actual movement of the prototype $\mathrm{Xu}$ the mechanical arm and the working space. The results show that the robot can well finish the work grasping foreign matter on the ground.
\end{abstract}

Keywords—robot; kinematic analysis; operation space analysis; reactor pool

\section{INTRODUCTION}

The rapid development of robot technology has greatly promoted the application of robots in various fields[1-4]. Especially in some special dangerous environments, it has become an indispensable equipment. At present, there are many problems in nuclear power plant environment, such as high radiation intensity, strong water corrosiveness, and many maintenance operations are difficult to finish[5]. In order to protect the safety of maintenance operators, robot technology has been widely accepted in nuclear industry[6].

\section{MULTI-FUNCTION UNDERWATER CRAWLING ROBOT SYSTEM FOR NUCLEAR POWER PLANT}

The robot adopts modular design, which is mainly composed of three parts, car body, manipulator and underwater camera, and the mechanical and electrical isolation between the parts is completely. Among them, six wheels equipped with camera and manipulator for underwater parts quickly arrived in working area, positioning observation and foreign unstructured working environment of radiation resistant underwater camera, mechanical hand for the completion of the salvage task of foreign bodies. By optimizing the relative position of each module, the robot is not only compact in structure but also can realize the omni-directional observation of the job site and the flexible grasping of the foreign objects. For specific environment and specific tasks, according to specific needs, we can selectively install components such as underwater camera, robot and other components, or adjust the installation position of the components. The typical combination form of robot is shown in Figure I.

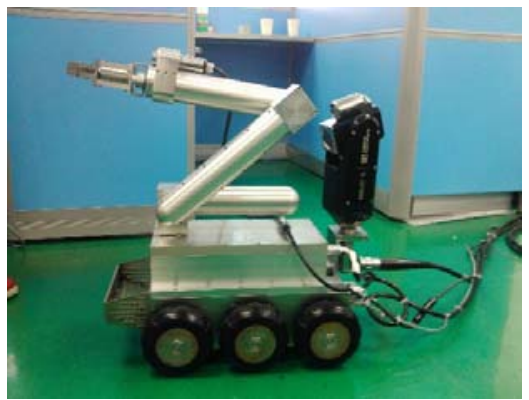

FIGURE I. THE TYPICAL COMBINATION FORM OF ROBOT

The robot control system has many advantages, such as versatile, reliable, practical and easy to operate. And focusing on the specific operation rules of nuclear power industry, the whole system has been optimized many times. The overall architecture is shown in Figure II. The control system adopts multi-sensor information fusion technology, which can comprehensively process underwater camera, small arm camera, robot driving state, robot posture and other information, and achieve accurate location of target foreign objects and accurate grasp of robot. The whole control system is divided into industrial control machine, hand control box, visual system and motion control system. The schematic diagram of its control system is shown in Figure II. 


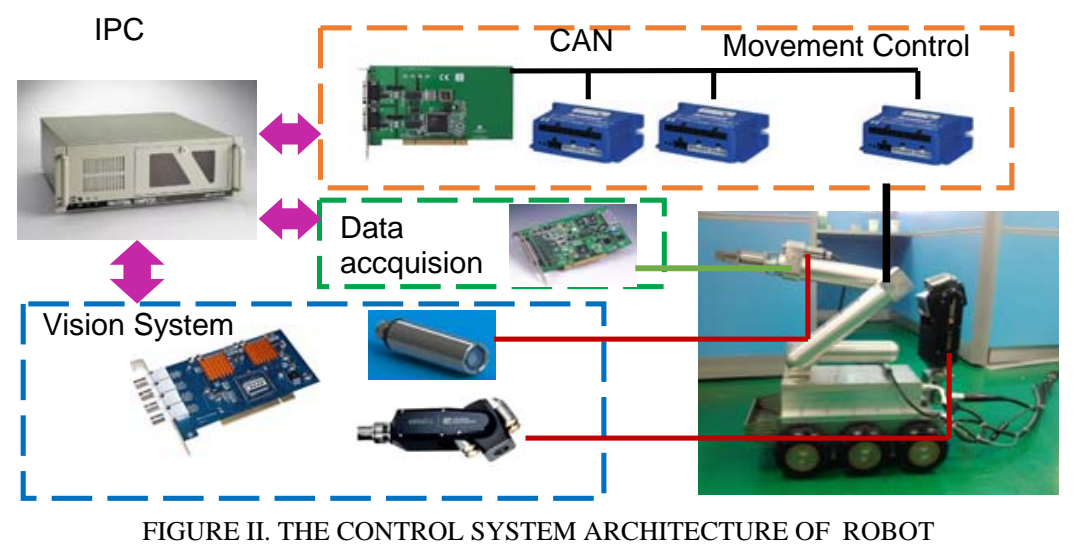

III. KINEMATICS ANALYSIS OF ROBOT

\section{A. Kinematics Positive Solution}

Figure III. shows the base coordinate system $\{\mathrm{S}\}$ and the tool coordinate system $\{\mathrm{T}\}$ for the initial configuration of a four degree of freedom manipulator.

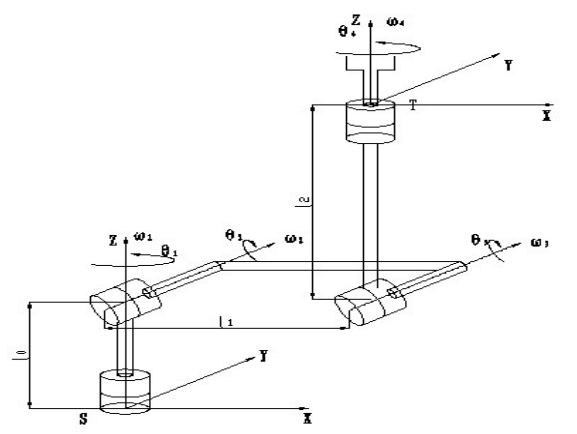

FIGURE III. THE ROBOT BASE COORDINATE SYSTEM

$$
g_{s t}(0)=\left(\begin{array}{cccc}
1 & 0 & 0 & l_{1} \\
0 & 1 & 0 & 0 \\
0 & 0 & 1 & l_{0}+l_{2} \\
0 & 0 & 0 & 1
\end{array}\right)
$$

The motion rotation coordinates of each joint are obtained by Eq. (1).

$$
\begin{aligned}
& \xi_{1}=\left(\begin{array}{c}
-\omega_{1} \times q_{1} \\
\omega_{1}
\end{array}\right)=\left(\begin{array}{llllll}
0 & 0 & 0 & 0 & 0 & 1
\end{array}\right)^{T} \\
& \xi_{2}=\left(\begin{array}{c}
-\omega_{2} \times q_{1} \\
\omega_{2}
\end{array}\right)=\left(\begin{array}{llllll}
-l_{0} & 0 & 0 & 0 & 1 & 0
\end{array}\right)^{T} \\
& \xi_{3}=\left(\begin{array}{c}
-\omega_{3} \times q_{2} \\
\omega_{3}
\end{array}\right)=\left(\begin{array}{llllll}
-l_{0} & 0 & l_{1} & 0 & 1 & 0
\end{array}\right)^{T} \\
& \xi_{4}=\left(\begin{array}{c}
-\omega_{4} \times q_{3} \\
\omega_{4}
\end{array}\right)=\left(\begin{array}{llllll}
0 & l_{1} & 0 & 0 & 0 & 1
\end{array}\right)^{T}
\end{aligned}
$$
(2).

The kinematics positive solution is obtained by the form Eq.

$$
g_{s t}(\theta)=\left(\begin{array}{cccc}
c_{1} c_{23} c_{4}-s_{1} s_{4} & -c_{1} c_{23} s_{4}-s_{1} c_{4} & c_{1} s_{23} & l_{1} c_{1} c_{2}+l_{2} c_{1} s_{23} \\
s_{1} c_{23} c_{4}+c_{1} s_{4} & -s_{1} c_{23} s_{4}+c_{1} c_{4} & s_{1} s_{23} & l_{1} s_{1} c_{2}+l_{2} s_{1} s_{23} \\
-s_{23} c_{4} & s_{23} s_{4} & c_{23} & l_{2} c_{23}-l_{1} s_{2}+l_{0} \\
0 & 0 & 0 & 1
\end{array}\right)
$$

\section{B. Inverse Kinematics of Manipulator}

When the kinematics is positive, the point on the axis of the joint $\quad q_{1}=\left(\begin{array}{lll}0 & 0 & l_{0}\end{array}\right)^{T} \quad, \quad q_{2}=q_{3}=\left(\begin{array}{lll}l_{1} & 0 & l_{0}\end{array}\right)^{T}$, $q_{4}=\left(\begin{array}{lll}l_{1} & 0 & l_{0}+l_{2}\end{array}\right)^{T}$ has been selected, and the unit vector on the axis is obtained $\omega_{1}=\left(\begin{array}{lll}0 & 0 & 1\end{array}\right)^{T}, \omega_{2}=\omega_{3}=\left(\begin{array}{lll}0 & 1 & 0\end{array}\right)^{T}$, $\omega_{4}=\left(\begin{array}{lll}0 & 0 & 1\end{array}\right)^{T}$.

In this paper, a kinematic rotation of pure rolling $\xi$ is used, and the position of any point on its axis $q_{\text {is constant, and the }}$ formula of the kinematic index product is simplified, that is $e^{\hat{\xi} \theta} q=q$.

$$
e^{\hat{\xi}_{1} \theta_{1}} e^{\hat{\xi}_{2} \theta_{2}} e^{\hat{\xi}_{3} \theta_{3}} e^{\hat{\xi}_{4} \theta_{4}} q_{4}=g_{s t} q_{4}
$$

$$
g_{s t} q_{4}=\left(\begin{array}{llll}
x & y & z & 1
\end{array}\right)^{T}
$$

$$
\begin{aligned}
& \text { From } e^{\hat{\xi} \theta} q=q \text {,it is known that: } \\
& \qquad e^{\hat{\xi}_{1} \theta_{1}} e^{\hat{\xi}_{2} \theta_{2}} e^{\hat{\xi}_{3} \theta_{3}} q_{4}=g_{s t} q_{4}
\end{aligned}
$$




$$
e^{\hat{\xi}_{1} \theta_{1}} e^{\hat{\xi}_{2} \theta_{2}} e^{\hat{\xi}_{3} \theta_{3}}=\left(\begin{array}{cccc}
c_{1} c_{23} & -s_{1} & c_{1} S_{23} & -l_{1} c_{1} c_{23}-l_{0} c_{1} S_{23}+l_{1} c_{1} c_{2} \\
s_{1} c_{23} & c_{1} & s_{1} S_{23} & -l_{1} S_{1} c_{23}-l_{0} S_{1} S_{23}+l_{1} s_{1} c_{2} \\
-S_{23} & 0 & c_{23} & l_{1} s_{23}-l_{0} c_{23}+l_{0}-l_{1} s_{2} \\
0 & 0 & 0 & 1
\end{array}\right)
$$

So,

$$
\left(\begin{array}{cccc}
c_{1} c_{23} & -s_{1} & c_{1} s_{23} & -l_{1} c_{1} c_{23}-l_{0} c_{1} s_{23}+l_{1} c_{1} c_{2} \\
s_{1} c_{23} & c_{1} & s_{1} s_{23} & -l_{1} s_{1} c_{23}-l_{0} s_{1} s_{23}+l_{1} s_{1} c_{2} \\
-s_{23} & 0 & c_{23} & l_{1} s_{23}-l_{0} c_{23}+l_{0}-l_{1} s_{2} \\
0 & 0 & 0 & 1
\end{array}\right)\left(\begin{array}{c}
l_{1} \\
0 \\
l_{0}+l_{2} \\
1
\end{array}\right)=\left(\begin{array}{c}
x \\
y \\
z \\
1
\end{array}\right)
$$

Suppose $S_{1}=x_{1}, C_{1}=x_{2}, S_{2}=x_{3}, C_{2}=x_{4}, S_{3}=x_{5}, C_{3}=x_{6}$, so

$$
\left\{\begin{array}{l}
p_{1}=x_{2} x_{3} x_{6} l_{2}+x_{2} x_{4} x_{5} l_{2}+x_{2} x_{4} l_{1}-x \\
p_{2}=x_{1} x_{3} x_{6} l_{2}+x_{1} x_{4} x_{5} l_{2}+x_{1} x_{4} l_{1}-y \\
p_{3}=x_{4} x_{6} l_{2}-x_{3} x_{5} l_{2}-x_{3} l_{1}+l_{0}-z \\
p_{4}=x_{1}^{2}+x_{2}^{2}-1 \\
p_{5}=x_{3}^{2}+x_{4}^{2}-1 \\
p_{6}=x_{5}^{2}+x_{6}^{2}-1
\end{array}\right.
$$

In the MMP platform, the computer language programming is carried out, and the $\mathrm{Wu}$ characteristic column of the polynomial equation group is obtained.

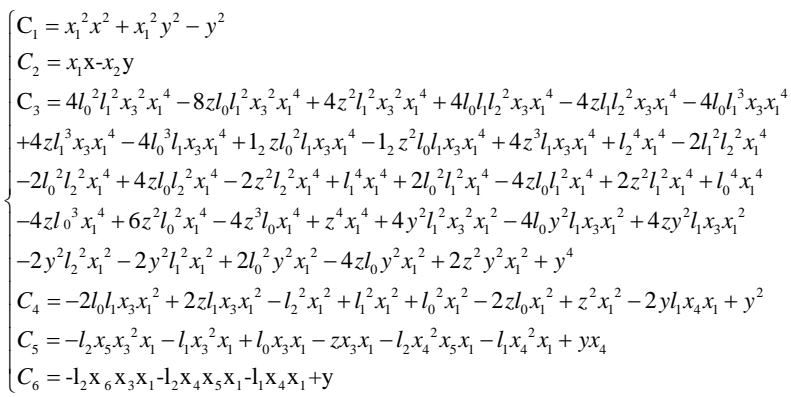

\section{ANALYSIS OF WORKING SPACE AND GROUND GRABBING SPACE OF ROBOT}

The underwater crawling robot of the nuclear power plant needs to predict the workspace and grasp the space, so as to accomplish the task with the help of remote operation. In this paper, the Monte Carlo method is used to calculate the workspace of the manipulator, and a visual working space "cloud picture" is obtained to understand the motion performance of the manipulator. Because of the manipulator to achieve the ultimate purpose of grasping objects on the ground, and the "cloud" can not accurately reflect the ground capture range, therefore, this paper analyzes the ground crawl space of the manipulator, the kinematics equation and the geometric equation of the surface side of each link has been established, the process of manipulator manipulator and constraint plane phase simultaneous, calculate the maximum ground crawl space boundary, Motion module using SolidWorks to simulate the boundary locus; using "robot simulation system" to record the interference check, the boundary feature points of different position, in order to obtain the minimum crawl space boundary, we can accurately grasp the ground space. The robot's working space "cloud map" and the ground grabbing space can not only reflect the space activity performance of the manipulator, but also accurately judge the scope of the ground grabbing, which lays the foundation for grasping planning.

\section{A. Ground Grabbing Space for Manipulator}

Because the manipulator ultimately wants to achieve the purpose of grasping the ground object. Before grasping, we should first determine whether the location is within the scope of the manipulator's surface grabbing, and the "cloud map" can not accurately reflect the scope of the ground grabbing. Therefore, by connecting the forward kinematics equation, the large arm and the small arm surface equation with the surface geometry equation and the ground constraint relation equation, the piecewise points are obtained, and the rotation angles of each joint and the maximum grasping boundary of the manipulator are obtained.

It is observed that the interference of the large arm and the body of the car and the interference of the end of the arm and the surface of the small arm occur in two cases when the four degree of freedom manipulator is grasping the ground foreign objects. First, the model of the large arm and the small arm is simplified. It is considered that the large arm and the small arm are cylindrical, and the circumferential surface equation of the big arm and the small arm is as follows:

$$
y^{2}+z^{2}-r^{2}=0
$$

$r$ is a circumferential radius,so,the equation of the cylindrical surface of the large arm and its edge in the world coordinate system:

$$
\left\{\begin{array}{l}
\left(l_{0} \cos \theta_{2}-z \cos \theta_{2}+x \cos \theta_{1} \sin \theta_{2}+y \sin \theta_{1} \sin \theta_{2}\right)^{2}+\left(l_{1}+y \cos \theta_{1}-x \theta_{1}\right)^{2}-r^{2}=0 \\
z \sin \theta_{2}-l_{0} \sin \theta_{2}-l_{1}+x \cos \theta_{1} \cos \theta_{2}+y \cos \theta_{2} \sin \theta_{1}=0
\end{array}\right.
$$

The equation of the circumferential surface of the small arm and the equation of the edge equation in the world coordinate system are:

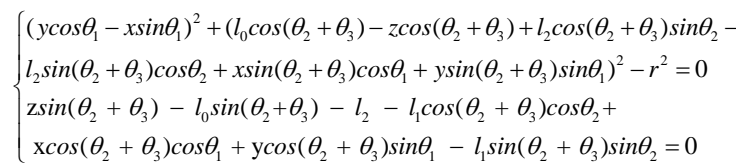


It is shown that the arm end edge with the platform of three edges, two end faces and the front camera platform two vertices of contact collision, establish each constraint equation, and the

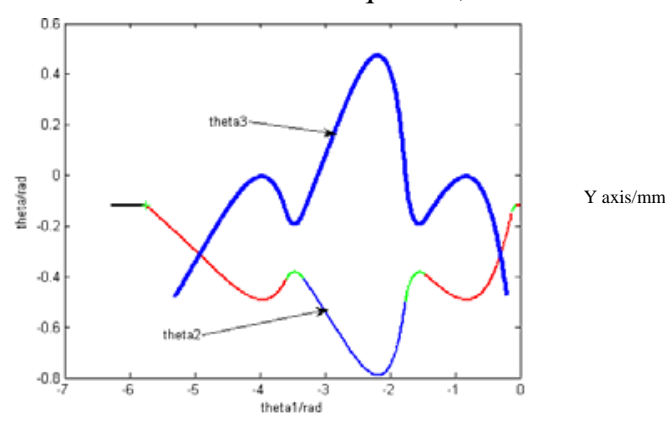

FIGURE IV. ANGLE CHANGE VALUE OF EACH JOINT

Using the trajectory generation and collision detection [7-8] in the Motion simulation module of Solidworks, we can get the spatial boundary curve of the manipulator's ground grabbing, as shown in Figure VI. From the diagram, the results are basically consistent with the results of theoretical analysis.

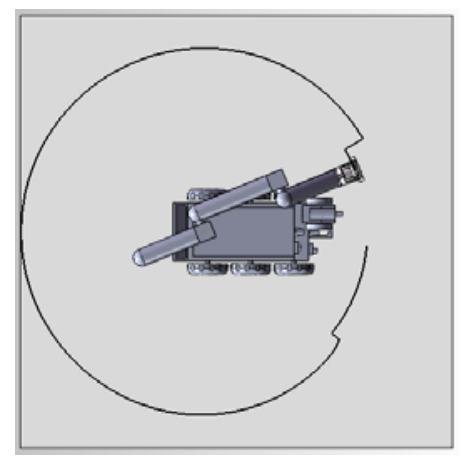

FIGURE VI. GROUND SIMULATION TRAJECTORY BASED ON SOLIDWORKS combination of forward kinematics equation and surface geometric equation, we can get the changing three angle and maximum ground crawl boundary, as shown in Figure IV V.

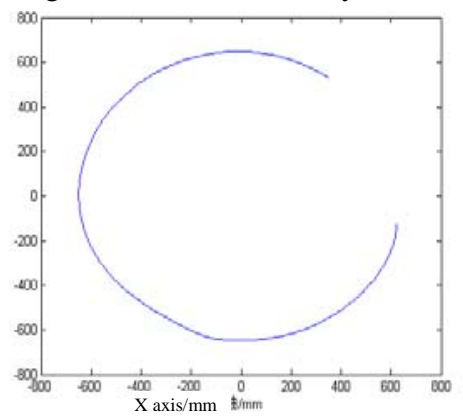

FIGURE V. GROUND CONTACT TRAJECTORY

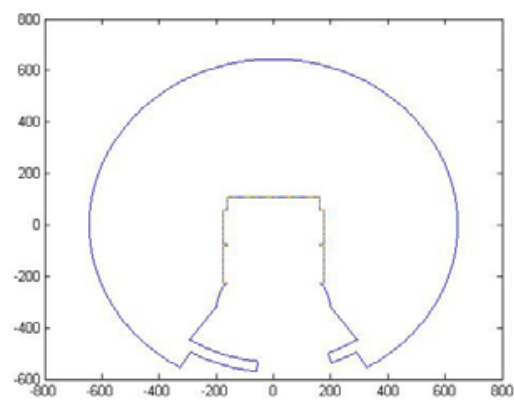

FIGURE VII. GROUND GRABBING SPACE FOR MANIPULATOR

According to the maximum boundary of the ground grabbing space has been obtained, for grabbing space, we should also care about whether or not we can grab near the car body, that is, the minimum boundary. In this paper, by using the simulation platform developed in the laboratory, the manipulator and the ground first establish constraints in different position, different positions of the manipulator with a small displacement of the second to interfere with the body just position, when the collision detection is just a collision, denoted as the termination point. The $\mathrm{X}, \mathrm{Y}, \mathrm{Z}$ coordinates of the point and the ground are recorded through the established database.

By repeating a large number of records, the minimum boundary of the grasping by the manipulator is obtained. The ground grabbing space of the manipulator is obtained by combining the maximum grabbing boundary and the minimum grasping boundary, as shown in Figure VIII. 


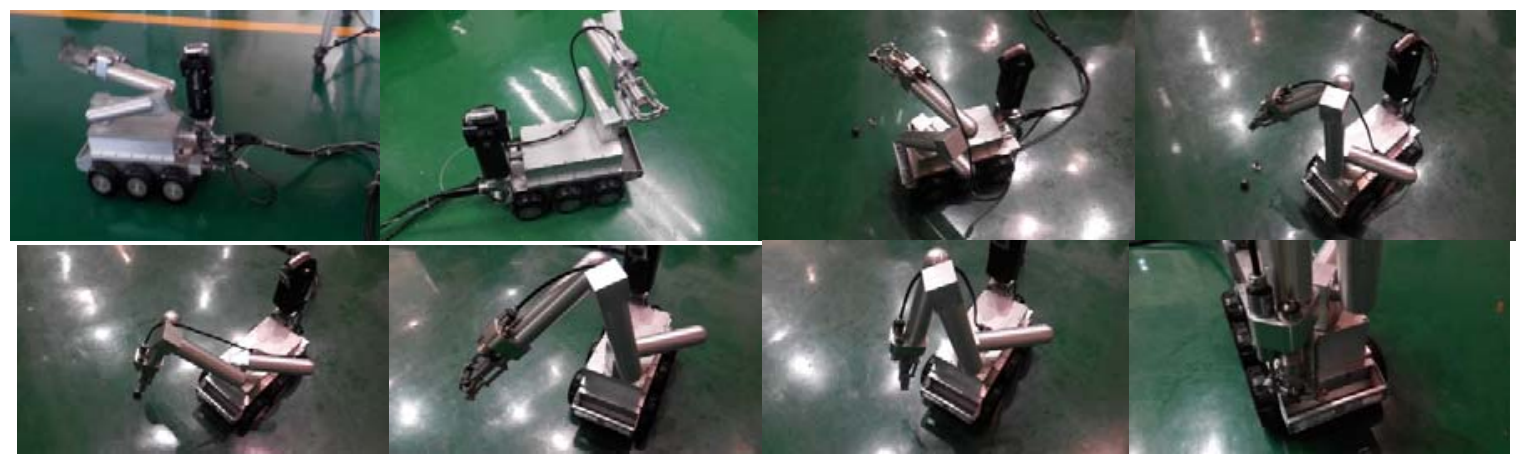

FIGURE VIII. PROTOTYPE GRASPING EXPERIMENT OF MULTI-FUNCTION UNDERWATER ROBOT IN NUCLEAR POWER PLANT

\section{CONCLUSION}

This paper is based on the study of the nuclear power plant multifunctional underwater robot kinematics m odel, and the forward and inverse solution equation, motion attitude control scheme so as to form the robot of the foreign body and salvage, and the kinematics equation of the manipulator and the geometric equation of surface equation, connecting rod of the manipulator and the constraint plane the combination of calculated maximum ground crawl space boundary, Motion module using SolidWorks to simulate the boundary locus, the simulation results show that the manipulator working attitude can be achieved on the bottom of the pool is convenient to grab. The parameters established by simulation are verified on the solid prototype. The working process is shown in the figure. The results show that the algorithm is accurate and the prototype is reliable.

\section{REFERENCES}

[1] Information on http://robot.ofweek.com

[2] S. U. Lee, Y. S. Choi, K. M. Jeong, and S. Jung, "Developmet of an underwater manipulator for maintaining nuclear power reactor," In International Conference on Control, Automation and Systems, Seoul, 2007, pp. 1006-1010.

[3] L. Wang, J. Wu, Y. Liu and D. Tang, "Desig and kinematics analysis of a robot for nuclear steam generator servicing,” Robot, vol. 31, no. 1, pp. 61-66, Jan. 2009.

[4] Ongena R, Ogawa Y. Nuclear power: Statusreport and future prospects [J]. Energy Policy, 2016, 96: 770-778.

[5] Keiji Nagatani,Seiga Kiribayashi,Yoshito Okada,Kazuki Otake,Kazuya Yoshida,Satoshi Tadokoro,Takeshi Nishimura,Tomoaki Yoshida,Eiji Koyanagi,Mineo Fukushima, Shinji Kawatsuma. Emergency response to the nuclear accident at the Fukushima Daiichi Nuclear Power Plants using mobile rescue robots[J]. J. Field Robotics. 2012 (1)

[6] Bruce Maclaurin. A skid steering model with track pad flexibility [J]. Journal of Terramechanics. 2006 (1)

[7] Nagatani K, Kiribayashi S, Okada Y, et al. Emergencyresponse to the nuclear accident at the fukushima daiichi nuclear power plants using mobile rescue robots [J]. Journal of Field Robotics, 2013, 30(1): 44-6

[8] Yang R, Clement B, Mansour A, et al.Modeling of a complex-shape underwater vehicle[C]//Autonomous Robot Systems and Competitions(ICARSC), 2014 IEEE International Conference on, IEEE, 2014: 36-41. 